\title{
Question mark ear deformity-revisited
}

\author{
Mohammad M. Al-Qattan ${ }^{1}$ - Noha M. Al-Qattan ${ }^{2}$
}

Received: 11 October 2016 / Accepted: 15 November 2016 / Published online: 29 November 2016

(C) Springer-Verlag Berlin Heidelberg 2016

\begin{abstract}
We report on two unusual cases of Cosman (question mark) ear; both required modifications of the standard techniques for surgical correction. The first patient presented with a unilateral question mark ear and concurrent ear prominence and bulging of the cartilage of the anti-helix. Simultaneous correction was done using a combination of cartilage suturing/scoring (for the prominence and the cartilage bulge) as well as Al-Qattan's "v-y skin flap-cartilage graft-z-plasty" technique (for the correction of the ear cleft deformity). The second patient had aurico-condylar syndrome with bilateral ear deformity and complete separation of ear lobes from the external ear. Staged transposition followed by Al-Qattan's technique resulted in a satisfactory outcome. Level of evidence: Level V, therapeutic study.
\end{abstract}

Key words Question mark · Ear · Aurico-condylar syndrome

\section{Introduction}

Cosman (question mark) ear is a congenital auricular cleft between the fifth and sixth hillocks [1-3]. The ear deformity is seen clinically in three different presentations: sporadic, familial (usually autosomal dominant) isolated ear deformity, and as a constant feature of aurico-condylar syndrome. Sporadic cases may be bilateral or unilateral [3]; while

Mohammad M. Al-Qattan

moqattan@hotmail.com

1 Division of Plastic Surgery, King Saud University, Riyadh, Saudi Arabia

2 Division of Plastic Surgery, National (Care) Hospital, Riyadh, Saudi Arabia inherited cases are almost always bilateral. Most patients with familial isolated ear deformities have mutations of the ENDOTHELIN-1 (EDN-1) gene [4]. Most patients with aurico-condylar syndrome have mutations of the PHOSPHOLIPASE C-Beta 4 (PLCB4) gene [5].

There are three main methods of surgical correction of question mark ears. The first technique utilizes a (skincartilage) composite graft to reconstruct the ear cleft defect [6]. In this technique, there are concerns regarding graft take and donor site morbidity. Several authors described cartilage grafts covered by various transposition skin flaps to the anterior surface of the ear $[2,7,8]$. These techniques provide adequate reconstruction but the anteriorly transposed skin flap introduces scars across the anterior surface of the ear. The third method was described by Al-Qattan [3] and has the main advantage of having minimal scarring on the anterior surface of the ear: the skin deficiency is corrected using a $v-y$ flap from the posterior auricular surface; the cartilage defect is reconstructed with a cartilage graft; and if needed, a z-plasty at the helical rim is done to realign the misdirected helix at the site of the cleft [3].

In this communication, we report in two unusual cases of question mark ear; both required modifications of the standard techniques for surgical correction.

\section{Case 1}

A 25-year-old male presented with unilateral question mark ear (Fig. 1a). Family history was negative. There was concurrent ear prominence, enlarged concha and bulging of the ear cartilage at the area the antihelix (Fig. 1a). Correction of the ear cleft was done using Al-Qattan ${ }^{3}$ technique: a v-y flap was advanced towards the helical rim to correct the skin deficiency (Fig. 1b, c); a cartilage graft (harvested from the ipsilateral concha) was used to reconstruct the rim cartilage deficiency; 
Fig. 1 Case Number 1. a The question mark ear with concurrent ear prominence and bulging of the cartilage at the upper part of the antihelix. b The longitudinal skin elliptical excision. The triangular flap will be advanced towards the rim. c The skin ellipse has been excised. The hooks hold the triangular flap. d Marking of the Z-plasty. e The Z-plasty flaps have been transposed and sutured. f Appearance at 3 months
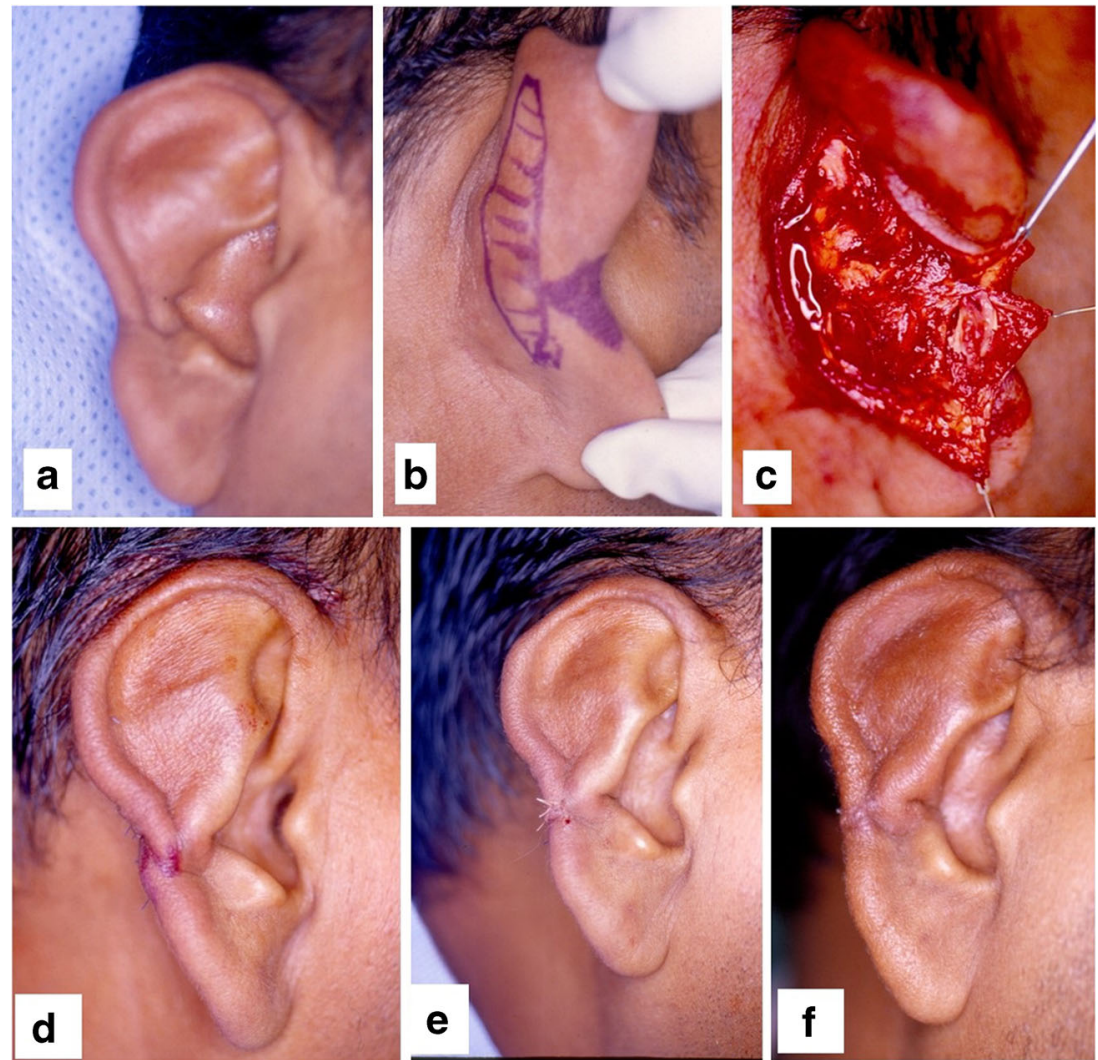

and finally, a Z-plasty was used to realign the misdirected helix at the cleft site (Fig. 1d, e). Harvesting the conchal cartilage graft helped to reduce the size of the concha. The cartilage bulge at the antihelix was corrected using anterior cartilage scoring. The prominence of the ear was also corrected simultaneously using a Mustarde technique by excision of a longitudinal strip of skin from the posterior aspect of the ear (posterior to the v-y flap as shown in Fig. 1b) and cartilage suturing to re-create an antihelix. The patient was satisfied with the outcome (Fig. 1 f).

\section{Case 2}

A 16-year-old girl presented with all the classic features of aurico-condylar syndrome (micrognathia, mandibular hypoplasia, prominent cheeks and bilateral question mark ears). The question mark ear deformity was very unusual because there was complete separation of the lobes from the external ear. Furthermore, the ear lobes were oriented vertically over the mastoids (Fig. 2a, b, Fig. 3a, b). Transposition of the ear lobes to the inferior aspect of external ear was done in two separate stages (4 weeks apart) (Fig. 2c, d, Fig. 3c, d). Both ears also had a very prominent antitragus which required reduction (Fig. 2c and Fig. 3c). Finally, the third stage was the utilization of the Al-Qattan ${ }^{3}$ technique to correct the residual cleft (Fig. 2e and Fig. 3e). The patient was satisfied with the outcome (Fig. 2f and Fig. 3f).

\section{Discussion}

The two cases of question mark ear presented in our report were unusual. The first case had concurrent prominence and we demonstrated that simultaneous correction of ear prominence and ear cleft reconstruction (using Al-Qattan technique) is possible and produces a satisfactory outcome in a single stage. The second case was unusual because of the complete separation of the ear lobes which required staged transposition of the ear lobes to the inferior aspect of the ears. Patients with aurico-condylar syndrome may have partial separation of the ear lobes [9]. Our case was unique because of the complete separation and the vertical orientation of the ear lobes and this made the reconstruction more challenging.

The pathogenesis of inherited question mark ears is well described in the genetics literature [10] and has not been discussed in the plastic surgery literature. The ears and the 
Fig. 2 Case Number 2. Right ear. a, b The deformity. Note the complete separation of the ear lobe. c Staged transposition of the ear lobe to the pinna. The prominent antitragus has also been reduced. d Appearance after ear lobe transposition (2 stages). e The residual ear cleft is small and hence the $v-y$ flap is small. A small cartilage graft will also be inserted under the flap. f Final appearance at 1 year
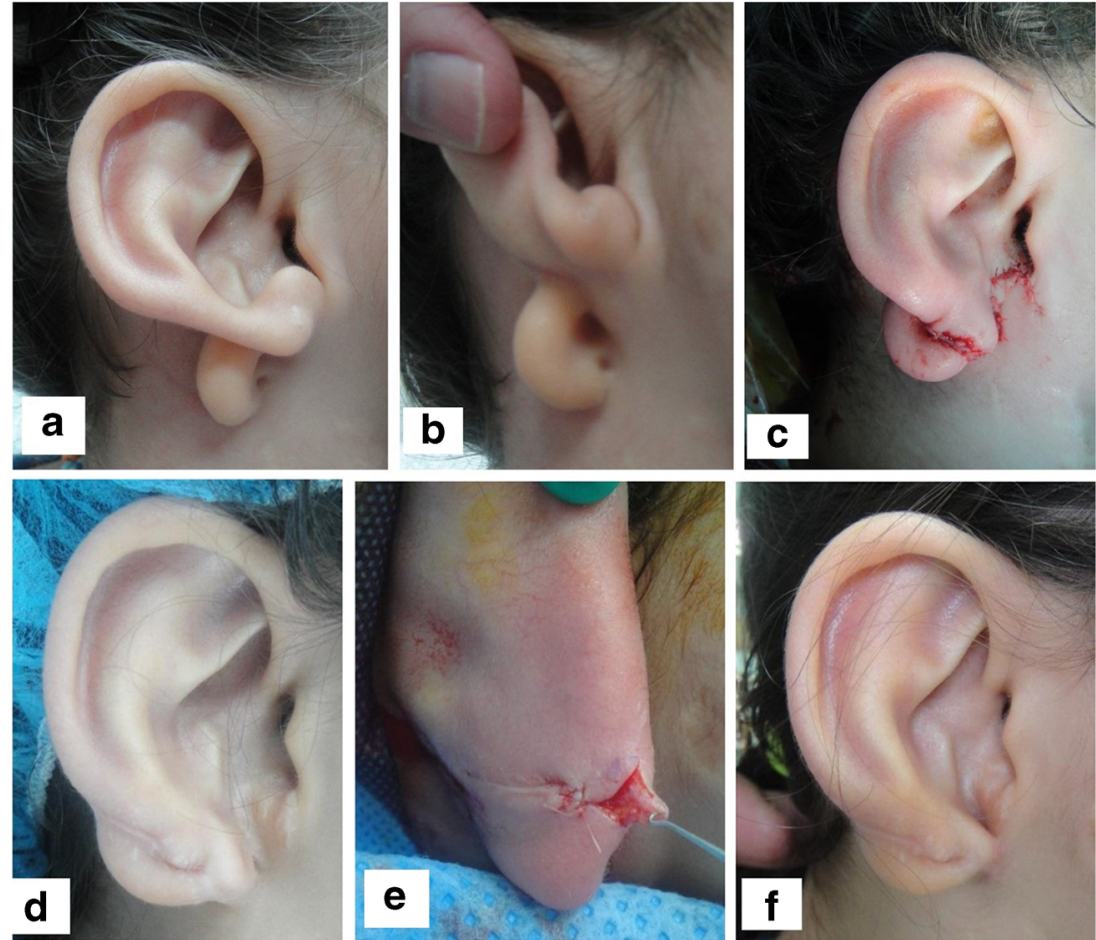

mandible develop from the branchial arches. Genetically, the regulation of facial morphogenesis through the brachial arches is mediated by "endothelin signaling". Endothelin signaling includes several proteins, receptors, and transcription factors. The most important proteins are Phospholipase C Beta
(PLCB) enzymes and Endothelin 1. PLCB enzymes regulate the development of neural crest cells for Endothelin 1. Endothelin 1 will then act through its receptor (Endothelin A receptor to control the development of the pharyngeal arches (including the ear and mandible) [10].
Fig. 3 Case Number 2. Left ear. a, b The deformity. c Staged transposition of the ear lobe to the pinna. d Appearance after ear lobe transposition (2 stages). e The residual cleft is large and hence a large $v$-y flap was raised and advanced towards the helical rim. f Final appearance at 1 year
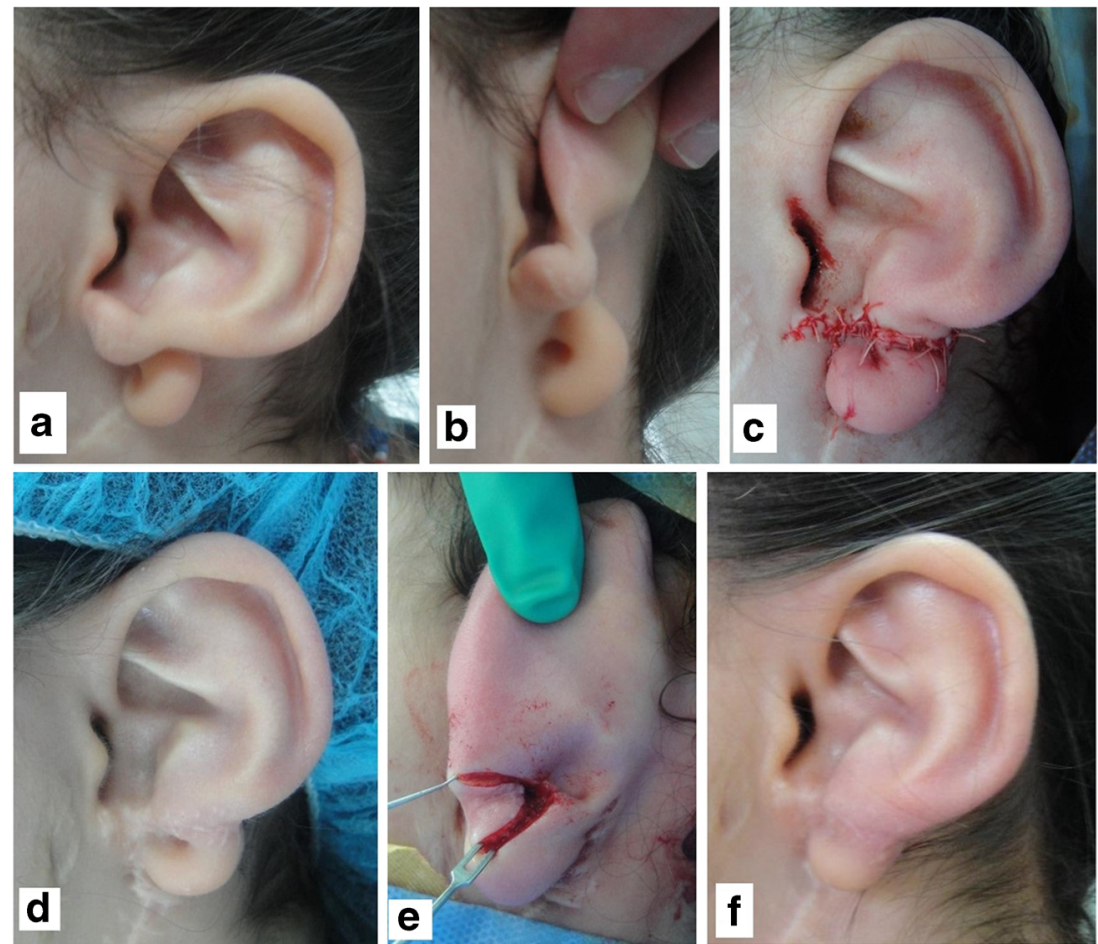


\section{Compliance with ethical standards}

Conflict of interest Mohammad M. Al-Qattan, Noha M. Al-Qattan declare that they have no conflict of interest.

Ethical approval For this type of study formal consent from a local ethics committee is not required.

Funding This work was supported by Al-Qattan and Noha College of Medicine Research Center, Deanship of Scientific Research, King Saud University, Riyadh, Saudi Arabia.

Patient consent Parents or legal guardians provided written consent before the inclusion of patients in this study. Additional consent was obtained for the use of their images.

\section{References}

1. Cosman B (1984) The question mark ear: an unappreciated major anomaly pf the auricle. Plast Reconstr Surg 73:572-576
2. Cosman B, Bellin H, Crikelair GF (1970) The question mark ear. Plast Reconstr Surg 46:454-457

3. Al-Qattan MM (1998) Cosman (question mark) ear: congenital; auricular cleft between the fifth and sixth hillocks. Plast Reconstr Surg 102:439-441

4. Gordon CT, Petit F, Kroisel PM et al (2013) Mutations in endothelin cause recessive auricocondylar syndrome and dominant isolated question-mark ears. Am J Hum Genet 93:1118-1125

5. Kido Y, Gordon CT, Sakazume S et al (2013) Further characterization of a typical features in auricocondylar syndrome caused by recessive PLCB4 mutations. Am J Med Genet 161A:2339-2346

6. Hall JD, Stevenson TR (1988) Congenital ear deformity: reconstruction using composite graft. Ann Plast Surg 21:145-148

7. Uysol OA, Kecik A, Gursu G (1990) Congenital auricular clefts. Eur J Plast Surg 13:178-181

8. Jia-Yi Z, Fen H (1996) Congenital auricular deformity consisting of cleft concha and transposition of the ear lobe and antitragus. Plast Reconstr Surg 97:428-430

9. McGowan R, Munday V, Kinning E et al (2011) Novel features in aurico-condylar syndrome. Clin Dysmorrph 20:1-6

10. Clouthier DE, Garcia E, Schilling TF (2010) Regulation of facial morphogenesis by endothelin signaling; insights from mice and fish. Am J Med Genet 152A:2962-2973 